\title{
Erratum: Efficient site-specific integration in Plasmodium falciparum chromosomes mediated by mycobacteriophage Bxb1 integrase
}

Louis J Nkrumah, Rebecca A Muhle, Pedro A Moura, Pallavi Ghosh, Graham F Hatfull, William R Jacobs Jr \& David A Fidock Nat. Methods 3, 615-621 (2006).

In the version of this article originally published, Figure $\mathbf{3 e - h}$ appears in black and white because of a production error. The color version

In addition, in the original version of Figure 3a, the pLN-ENR-GFP plasmid was incorrectly labeled with attB when the black box should indicate attP. The errors have been corrected in the HTML and PDF versions of the article. 